risk of GIB. These findings are in contrast to the current practice where endoscopic biopsies are usually not done at platelet counts lower than the 20,000 or 50,000 cells/cc and there is generally reluctance to perform endoscopic interventions in patients with thrombocytopenia questioning their efficacy in situations where normal hemostatic mechanisms of body are impaired. Also, it is important to remember that relying only on platelet counts is not correct as an important determinant of hemostasis is the platelet function and patients may bleed despite near normal platelet counts because of impaired platelet functions. The authors thus concluded that aiming for platelet counts of more than 50,000/cc before endoscopy is not necessary and platelet count more than $25,000 / \mathrm{cc}$ are sufficient for endoscopic biopsies, and platelet count more than 40,000 are reasonable for removal of small ( $<10 \mathrm{~mm}$ ) polyps with modest risk of bleed.

\section{Assessment of Crohn's disease activity by confocal laser endomicroscopy}

\begin{abstract}
Helmut Neumann, Michael Vieth, Raja Atreya, Martin Grauer, Jurgen Siebler, Thomas Bernatik, Markus F. Neurath, and Jonas Mudter
\end{abstract}

Inflamm Bowel Dis 2012;18:2261-2269

Endoscopy is the standard of care for diagnosing as well as assessing the extent and activity of inflammatory bowel diseases. ${ }^{[5,6]}$ The endoscopic changes visible on white light may lag behind the histopathological changes and therefore microscopic examination of the endoscopic biopsies is done to assess the histological activity. Confocal laser endomicroscopy (CLE) is a new upcoming diagnostic modality that has the potential of a real-time in vivo histopathology after application of exogenous fluorescence agent with a 1000 times magnification. ${ }^{[7]}$ CLE has been shown to be a useful diagnostic modality in ulcerative and microscopic colitis but its role in Crohn's disease $(\mathrm{CD})$ is not clear. ${ }^{[8,9]}$

In this prospective study 27 patients with quiescent and 27 patients with active CD were studied (mean age 39 years; $52 \%$ females) and were compared 18 controls (mean age: 68 years and $50 \%$ females). The CD activity in these patients was assessed using CD Endoscopic Index of Severity. The colonoscopy was performed by expert endoscopist and CLE was performed under conscious sedation using either an integrated endoscopy system (iCLE; Pentax) or a probe-based system (pCLE, Cellvizio, Mauna Kea Technologies, Paris, France). iCLE images were taken at a frame rate of $0.8 / \mathrm{s}$ at $1024 \times 1024$ pixels or $1.6 / \mathrm{s}$ at $1024 \times 512$ pixels. The normal mucosa and pathological lesions were evaluated as per Mainz Journal of Digestive Endoscopy Vol 5 | Issue 2 | April-June 2014 confocal pattern classification for iCLE and as per Miami classification for pCLE. ${ }^{[10,11]}$ After performing CLE, endoscopic biopsies were taken from both macroscopically normal and abnormal mucosa and findings of the confocal images were compared with the histology findings.

\section{Confocal Laser Endomicroscopy Findings in Controls}

All these controls had regular colonic architecture with normal crypts and microvascular pattern revealed normal vessel structures without fluorescein leakage or microerosions. There were no signs of increased cellular infiltrates within the lamina propria and the goblet cells were regularly shaped.

\section{Confocal Laser Endomicroscopy Findings in Quiescent Crohn's Disease}

Patients of CD had significantly increased number of colonic crypts (67\% vs. $11 \%$, respectively, $P=0.001)$, increased crypt tortuosity (52\% vs. $11 \%$, respectively, $P=0.02$ ), and dilated crypt lumens (52\% vs. $6 \%$, respectively, $P=0.008)$ compared with the controls. The frequency of increased vascularity $(44 \%$ vs. $6 \%$, respectively, $P=0.027)$ and an increased number of goblet cells (63\% vs. $6 \%$, respectively, $P<0.001$ ) was also more in patients of $\mathrm{CD}$ when compared to controls. However, there was no significant difference between the patients and controls in the presence of micro-erosions and cellular infiltrates within the lamina propria. Increased number of goblet cells on multivariate analysis was found to be the best predictor for quiescent CD (odds ratio, 28.9; 95\% confidence interval: 4.5-186.1).

\section{Confocal Laser Endomicroscopy Findings in Active Crohn's Disease}

The patients with active $C D$ were found to be having significantly decreased number of colonic crypts $(93 \%$ vs. $6 \%$, respectively, $P<0.001$ ), increased crypt tortuosity ( $100 \%$ vs. $0 \%$, respectively, $P<0.001)$, dilated crypt lumen $(100 \%$ vs. $17 \%$, respectively, $P<0.001)$, more microerosions $(100 \%$ vs. $0 \%$, respectively, $P<0.001)$, increased cellular infiltrate $(100 \%$ vs. $0 \%$, respectively, $\mathrm{P}<0.001)$, increased vascularity $(100 \%$ vs. $11 \%$, respectively, $P<0.001)$, and decreased goblet cells $(85 \%$ vs. $6 \%$, respectively, $P<0.001)$ in comparison to controls. The interobserver agreement ranged from moderate (crypt lumen, microerosions, goblet cell number) to substantial (tortuosity of crypts, vascularity) to almost perfect (number of colonic crypts, cellular infiltrate) with highest kappa-values for interobserver and intraobserver agreement being found for the number of colonic crypts ( 0.943 and 0.950 , respectively).

On the basis of these findings the authors proposed $C D$ Endomicroscopic Activity Score (CDEAS) that consists of six parameters: Crypt number (increased or decreased), crypt distorsion, microerosions, cellular infiltrate, vascularity, and 
number of goblet cells (increased or decreased) with one point for each parameter and thus the score ranging from 0 to 8 . They found that CDEAS strongly correlated with C-reactive protein values and the mean CDEAS scores were 2 and 5 in patients with quiescent and active $C D$ respectively $(P<0.001)$. The authors concluded that the CLE is able to predict disease severity even in quiescent $C D$ with high kappa values and accuracy and therefore is more sensitive than standard whitelight endoscopy in patients with CD.

\section{Commentary}

Although endoscopy has revolutionized the management of various GI disorders like inflammatory bowel diseases but it has certain limitations also. Inability to diagnose early or mild lesions that have subtle or no changes on white light endoscopy is the major limitation of standard endoscopy and in these situations one has to rely on histopathology for correct diagnosis. Ability to perform real time in vivo histopathology would be a dream come true for the endoscopist and CLE has the potential to fulfill this aspiration of endoscopist. In this study, the authors for the first time systematically used CLE to determine the disease activity in CD and have elegantly shown that it could identify architectural changes in active CD similar to the established histopathological criteria. They demonstrated an added advantage of real time histology whereby an increased vascularity was found to be typical for active $C D$ but not quiescent $C D$ and this finding is not seen on routine histopathology. The most important finding of this study was that CLE was able to differentiate between quiescent $\mathrm{CD}$ with macroscopic normal mucosa and controls and number of colonic segments without macroscopic inflammation on colonoscopy had histological and CLE evidence of inflammation. The findings of this study are novel and have potential to change our endoscopic practice but further studies are needed to ascertain if the activity assessment of CD by CLE will have a better predictive value for predicting the course of disease and response to therapy.
Surinder Singh Rana

Department of Gastroenterology, Post Graduate Institute of Medical Education and Research, Chandigarh, India

Address for correspondence: Dr. Surinder Singh Rana, Department of Gastroenterology, Post Graduate Institute of Medical Education and Research, Chandigarh - 160 012, India. E-mail: drsurinderrana@yahoo.co.in

\section{Suggested Reading}

1. Schiffer CA, Anderson KC, Bennett CL, Bernstein S, Elting LS, Goldsmith $\mathrm{M}$, et al. Platelet transfusion for patients with cancer: Clinical practice guidelines of the American society of clinical oncology. J Clin Oncol 2001;19:1519-38.

2. Ponec RJ, Hackman RC, McDonald GB. Endoscopic and histologic diagnosis of intestinal graft-versus-host disease after marrow transplantation. Gastrointest Endosc 1999;49:612-21.

3. Van Os EC, Kamath PS, Gostout CJ, Heit JA. Gastroenterological procedures among patients with disorders of hemostasis: Evaluation and management recommendations. Gastrointest Endosc 1999;50:536-43.

4. Rebulla P. Platelet transfusion trigger in difficult patients. Transfus Clin Biol 2001;8:249-54.

5. Annese V, Daperno M, Rutter MD, Amiot A, Bossuyt P, East J, et al. European evidence based consensus for endoscopy in inflammatory bowel disease. J Crohns Colitis 2013;7:982-1018.

6. Sreerama RK, Chhabra P, Sharma V. Clinical, endoscopic and histopathological parameters at presentation that predict need for longterm immunosuppression in ulcerative colitis. J Dig Endosc 2014. [In press].

7. Neumann H, Kiesslich R, Wallace MB, Neurath MF. Confocal laser endomicroscopy: Technical advances and clinical applications. Gastroenterology 2010;139:388-92.e1.

8. Li CQ, Xie XJ, Yu T, Gu XM, Zuo XL, Zhou CJ, et al. Classification of inflammation activity in ulcerative colitis by confocal laser endomicroscopy. Am J Gastroenterol 2010;105:1391-6.

9. Kiesslich R, Hoffman A, Goetz M, Biesterfeld S, Vieth M, Galle PR, et al. In vivo diagnosis of collagenous colitis by confocal endomicroscopy. Gut 2006;55:591-2.

10. Kiesslich R, Burg J, Vieth M, Gnaendiger J, Enders M, Delaney P, et al. Confocal laser endoscopy for diagnosing intraepithelial neoplasias and colorectal cancer in vivo. Gastroenterology 2004;127:706-13.

11. Wallace M, Lauwers GY, Chen Y, Dekker E, Fockens P, Sharma P, et al. Miami classification for probe-based confocal laser endomicroscopy. Endoscopy 2011;43:882-91. 